\title{
Assessment of Immunomodulatory Effect on Natural Antioxidants - An In Vitro Study
}

\author{
Deepigaa Manivasagam ${ }^{1}$, Arvind Muthukrishnan², S. Rajeshkumar ${ }^{3}$
}

1, 2, 3 Department of Oral Medicine and Radiology, Saveetha Institute of Medical and Technical Sciences (SIMATS), Kuthambakkam, Tamil Nadu, India.

\section{ABSTRACT}

\section{BACKGROUND}

Garlic has anti allergic, anti-tumour and chemo preventive effect. Turmeric has analgesic, anti-inflammatory, wound healing, and immunomodulatory activities. Spirulina has antioxidant, immunomodulatory, anti-inflammatory, anticancer, antiviral, and antibacterial effects. Honey has antimicrobial, antioxidant, anticancer, antiinflammatory. The purpose of this study was to prepare herbal formulations of garlic, turmeric, honey, and spirulina and analyse its immunomodulatory effect.

\section{METHODS}

Garlic, turmeric, honey, and spirulina were combined with $100 \mathrm{ml}$ of distilled water and simmered for 15 minutes before being filtered and condensed again to $5 \mathrm{ml}$. The diphenyl 1-picryl-hydrazyl-hydrate test (DPPH), ferric reducing antioxidant power assay (FRAP) and H2O2 assays were used to evaluate the immunomodulatory effect.

\section{RESULTS}

The prepared extract showed significant results at $50 \mathrm{l}$ when assessed using DPPH, FRAP and $\mathrm{H} 2 \mathrm{O} 2$ assay (i.e.) $72.1 \%, 94.6 \%$ and $67.9 \%$ respectively.

\section{CONCLUSIONS}

This in-vitro study shows that the combination of garlic, turmeric, honey and spirulina formulation have good immunomodulatory effects when compared to the standard. Hence, in future, it can be used as a safe alternative instead of commercially available medicines in the form of in-situ gel for management of immune mediated disorders.
Corresponding Author: Dr. Arvind Muthukrishnan, 162, Poonamalle High Road, Velappanchavadi, Tamil Nadu, India. E-mail: arvindm@saveetha.com

DOI: $10.14260 / \mathrm{jemds} / 2021 / 733$

How to Cite This Article: Manivasagam D, Muthukrishnan A, Rajeshkumar S. Assessment of immunomodulatory effect on natural antioxidants - an in vitro study. J Evolution Med Dent Sci 2021;10(42):3612-3616, DOI: $10.14260 /$ jemds/2021/733

Submission 25-03-2021, Peer Review 29-05-2021, Acceptance 05-06-2021, Published 18-10-2021.

Copyright (C) 2021 Deepigaa Manivasagam et al. This is an open access article distributed under Creative Commons Attribution License [Attribution 4.0 International (CC BY 4.0)]

\section{KEY WORDS}

Garlic, Turmeric, Honey, Spirulina, Antioxidant, Immunomodulator 


\section{BACKGROUND}

Immunomodulation is a change in immune reaction that either decreases or increases immune response. Immunostimulation is the process of increasing immune response, whereas immunosuppression is the process of decreasing immune responsiveness. A substance that stimulates, suppresses, or modulates any part of the immune response, which include both adaptive and innate immune system is called as immunomodulators. ${ }^{1}$ The essence of immunomodulation depends on the immunomodulating effect of pharmacological agents acting under various doses and time.,3 Majorly used immunomodulators are natural adjuvants, synthetic agents, antibody reagents. As they have generalized effects throughout the immune system, a lot of adverse effects have been reported. ${ }^{4}$ In order to overcome the side effects, variety of drugs derived from natural sources, either plants or minerals, are used as an alternative to modify the immune system. ${ }^{5}$ There are many other natural products that are used in various medical systems around the world to treat immunological disorders. Plant products have been used successfully in the management of a variety of diseases since $6000 \mathrm{BC}$ in India, 4,6

Allium sativum is an excellent nutrient component that is primarily grown in India and is commonly known as garlic around the world. Garlic has been shown to have immunomodulatory effects in vitro and in vivo studies, with increased T-lymphocyte blastogenesis and phagocytosis, as well as activation of cytokine production. Aged garlic extract has demonstrated various anti-tumour and anti-allergic effects. ${ }^{7}$ There was significant reduction of IL-12 production and increase in IL-10 production with low concentration of garlic extract. ${ }^{8}$ It is also reported that with the extract, there is significant reduction with TNF - $\alpha$ (tumour necrosis factor), IL - 1 $\alpha$, IL - 6, IL - 8, T cell interferon - gamma (IFN - $\gamma$ ), IL - $2 .{ }^{9}$

Curcuma longa is a perennial herb that is widely grown in India. C. longa rhizome has variety of medicinal uses, which include analgesic, anti-inflammatory, wound healing, and immunomodulatory properties. ${ }^{10}$ Curcumin, the main constituent of $C$. longa, is important for immunomodulatory activity. ${ }^{11}$ Curcumin administration increased bone marrow cellularity, alpha-esterase positive cells, and macrophage phagocytic activity. ${ }^{12}$ Numerous studies suggest that curcumin can influence both $\mathrm{T}$ cell proliferation and activation. ${ }^{13}$ Curcumin has been shown to decrease the proliferation of $\mathrm{T}$ lymphocytes isolated from healthy donors induced by phorbol myristate acetate (PMA) and anti-CD28 antibodies, as well as that caused by phytohemagglutinins (PHA). ${ }^{14}$ It can also reduce IL-2 expression and NF-B39 expression, as well as reduce phytohemagglutinin-induced proliferation of human peripheral blood mononuclear cells. ${ }^{15}$ The processes of honey's immunomodulatory properties were unclear, but a few authors speculated that high concentration of hydrogen peroxide in honey might have triggered a negative feedback effect on MM6 cell ROI production. ${ }^{16}$ Increased TNF- $\alpha$, interleukin-1 (IL 1 $1 \beta$ ) and IL-6 cytokines were also reported with cells of MM6 and with human blood monocytes. ${ }^{17}$

Spirulina, a cyanobacteria from Oscillators, has been a source of protein and vitamins since time immemorial. ${ }^{18}$ The most common species used in the field of medicine are Spirulina platensis and Spirulina maxima. ${ }^{19}$ Spirulina has proven to be a powerful stimulant of the immune system through the increased stimulation of IL-1 $\beta$, IL-4 and interferon. ${ }^{20}$ Spirulinas have proved to be antioxidant, antiinflammatory, anti-cancer, anti-virus and antibacterial. Increasing the expression of TNF- $\alpha$, IL- $1 \beta$ and IL- 6 cells with expression of IL-1ßen and COX-2 proteins have been shown to be phycocyanins which are a component of spirulina. ${ }^{21}$

Thus, the aim of this study was to perform in vitro analysis to assess the combined immunomodulatory effect of garlic, turmeric, honey, and spirulina.

\section{METHODS}

This in vitro study was conducted from November 2020 to March 2020 and materials used in this study include extracted compounds containing $1 \mathrm{~g}$ of each herbal, garlic, turmeric, honey, and spirulina; these materials were acquired from authentic biomaterial sellers. A beaker of $100 \mathrm{~mL}$ of distilled water was taken and extract of $1 \mathrm{~g}$ garlic, turmeric, honey and spirulina were mixed together. These extracts were then heated for 15 minutes, filtered and again heated until the solution was reduced to about $5 \mathrm{~mL}$ of concentration, in order to reduce the water and increase the concentration of extraction followed by cooling down of the prepared extract [Figure 1].

The prepared extract of garlic, turmeric, honey and spirulina is subjected to 2, diphenyl 1-picryl-hydrazyl- hydrate assay (DPPH). The extract was first subjected to 5 different concentrations of $10 \mu \mathrm{L}, 20 \mu \mathrm{L}, 30 \mu \mathrm{L}, 40 \mu \mathrm{L}, 50 \mu \mathrm{L}$ in solution containing $1 \mathrm{~mL}$ of DPPH. The solution was maintained at room temperature for 10 minutes, followed by boiling of the contents at 55 degree Celsius for 10 - 15 minutes. This solution is then subjected to spectrophotometry for inhibition level analysis. The reduction in the quantity of DPPH free radicals was assessed dependent on the absorbance at $517 \mathrm{~nm}$ [Figure 2a].

Ferric reducing antioxidant power assay (FRAP) was then performed. FRAP solution ( $3.6 \mathrm{~mL}$ ) was added to distilled water $(0.4 \mathrm{ml})$ and incubated at $37 \mathrm{C}$ for $5 \mathrm{~min}$. Then this solution was mixed with $10 \mu \mathrm{L}, 20 \mu \mathrm{L}, 30 \mu \mathrm{L}, 40 \mu \mathrm{L}, 50 \mu \mathrm{L}$ concentration of prepared extract of garlic, turmeric, honey and spirulina and incubated at 37C for 10 minutes. The absorbance of reaction mixture was measured at $593 \mathrm{~nm}$ [Figure 2b].

H2O2 assay was performed by Halliwell method. All solutions were prepared freshly $1.0 \mathrm{~mL}$ of the reaction mixture contained $100 \mathrm{~L}$ of $28 \mathrm{mM}$ of 2-deoxy-2-ribose (dissolved in phosphate buffer; $\mathrm{pH}$ - 7.4), $500 \mathrm{~L}$ solution of various concentration of the extract $(10-50 \mathrm{~L}), 200 \mathrm{~L}$ of $200 \mathrm{~m} \mathrm{Fecl} 3$ and $1.04 \mathrm{mM}$ EDTA $(1: 1 \mathrm{v} / \mathrm{v}), 100 \mathrm{~L} \mathrm{H} 2 \mathrm{O} 2(1.0 \mathrm{mM})$ and 100 $\mathrm{L}$ ascorbic acid (1.0 mM). After an incubation period of 1 hour at $37 \mathrm{c}$, the extent of deoxyribose degradation was measured by TBA reaction and the absorbance was measured at $532 \mathrm{~nm}$ [Figure 2c].

\section{Statistical Analysis}

Statistics was done using IBM Statistical Package for Social Sciences (SPSS 23). $\mathrm{t}$ test was performed to assess the 
statistical significance between the assay group and control groups.

\section{RESULTS}

The results of this study have shown that the prepared extract of garlic, turmeric, honey and spirulina have better inhibition action in DPPH, FRAP, $\mathrm{H} 2 \mathrm{O} 2$ assay analysis. Spectrophotometry readings of DPPH, FRAP, H2O2 assay shows that the absorbance range was close to the standard (vitamin C) and also at 50 of garlic, turmeric, honey and spirulina have more absorption percentage of $72.1 \%$, $94.6 \%$ and $67.9 \%$ respectively [Figure 3,4 and 5]. Statistically significant difference was observed between the assay group and standard group [table 1]

\begin{tabular}{|cccc|}
\hline Groups & Mean & $\begin{array}{c}\text { Standard } \\
\text { Deviation }\end{array}$ & P Value \\
Standard & 84.50 & 6.93 & - \\
DPPH assay & 66.76 & 4.93 & 0.000 \\
FRAP assay & 67.46 & 20.93 & 0.002 \\
H202 assay & 49.08 & 11.55 & 0.001 \\
\hline \multicolumn{4}{c}{ Table 1 Statistical Difference between } \\
& Standard Group and Assay Group Using t Test. \\
\hline
\end{tabular}
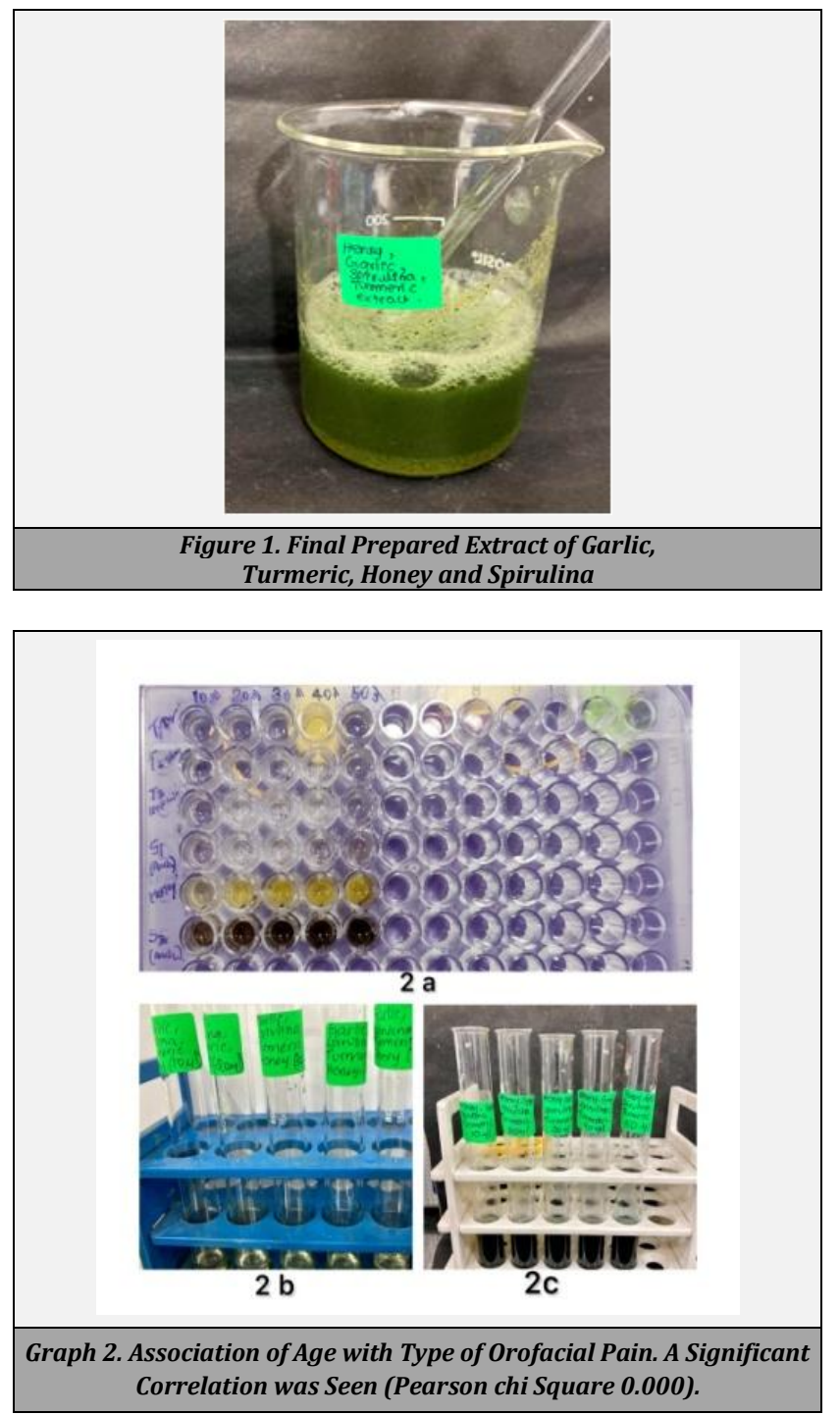

DPPH Assay

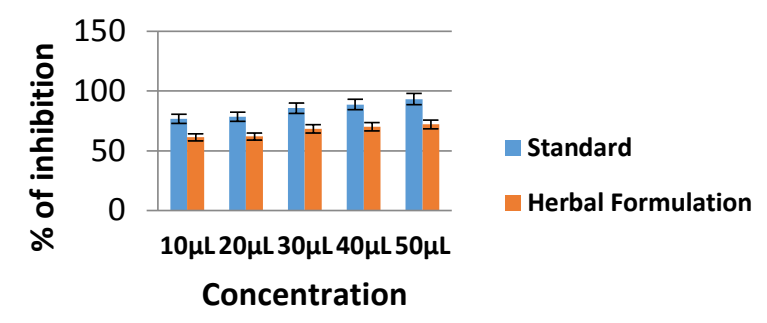

Figure 3. Using DPPH Assay, the Absorbance of Reaction Mixture Measured at $517 \mathrm{~nm}$
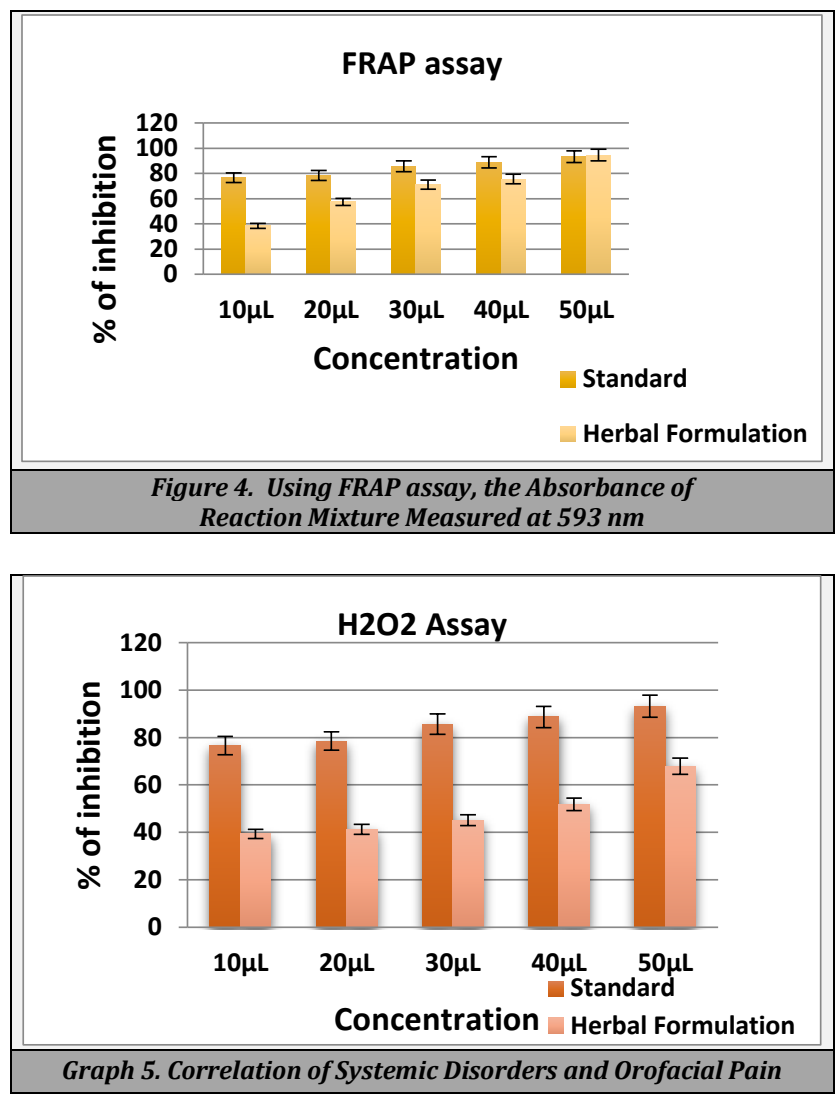

\section{DISCUSSION}

Allium plants have an interesting biological and pharmacological properties because of their presence of organosulfur compounds. One of the most commonly used among these is garlic (Allium sativum). These compounds exhibit large range of benefits against microbial infections and are well known for their cardioprotective, anticancer, immunomodulatory and anti-inflammatory activities when extracted and isolated. ${ }^{22}$ Inflammatory conditions related to inflammatory medications i.e. induction, oxidative stress and activation of different immune cells is the reason for the effect of garlic on immune systems components. ${ }^{8}$ The candidate is promising to keep the immune system homeostasis. ${ }^{23}$ Thiacremonone, an isolated sulphur compound in garlic blocks NFB activity in in vitro and improves inflammatory responses and arthritis in acute and chronic oedema in arthritis animal models, have been found to inhibit iNOS and 
NO" production, as reported by Ban J et al. ${ }^{24}$ The antioxidant effect of the cellular antioxidant enzymes, like superoxide dismutase, catalase and glutathon peroxidase and important source of antioxidants, was reported by Badr $\mathrm{G} M$ et al. who are an important source of antioxidants from phytochemicals like DAS and SAMC. ${ }^{25}$ A study by You H.S et al. shows that the garlic extract suppressed the dimerization of the LPS-induced TLR4, indicating that it is one of the anti-inflammatory garlic ${ }^{23}$ mechanisms. Through the suppression of TLR activation leading to an activation of NF ÚB and COX - 2 and iNOS expression inhibition, garlic may thus modulate inflammatory responses.

Turmeric constituents include three curcuminoids such as curcumin, dimethoxy curcumin and bisde-methoxy curcumin. Curcumin has the ability to suppress cellular transformation, proliferation, invasion, angiogenesis and metastasis ${ }^{26}$ Khar A et al. reported that tumour cell lines immune to curcuminmediated apoptosis, dependent on intermediate ROS, have been induced by stress response. ${ }^{27}$ The dose of curcumin establishes ROS, intracellular adenosine triphosphate (ATP), apoptosis or osteoblast necrosis. ${ }^{28}$ In a study done by Limtrakul et al. curcumin modulates the reversal of multi-drug resistance. ${ }^{29}$ Curcumin plays a direct role in the treatment of varied autoimmune disorders and it inhibits IL-12-mediated neuronal Th1 dependent demyelination by aiming Janus kinase 2, tyrosine kinase 2, STAT3, and STAT4 on the murine model of multiple sclerosis as reported by Natarajan C et al. ${ }^{30}$ This also improves the clearance of amyloid- $\beta$ (plaques) by M $\mu$ s (macrophages) within the brain in patients with Alzheimer's disease. ${ }^{31}$ Curcumin attenuated LPS-mediated endotoxemia in severe conditions of infection. ${ }^{32}$ Curcumin is aimed to achieve an anti-inflammatory response of TLRadapter-MD- 2 and to inhibit homodimerization of TLR4. ${ }^{33,34}$ Cho JW et al. reported in a study that curcumin has been found to modulate human keratinocytes treated with TNF- $\alpha$ expression of IL-1 $\beta$, IL-6, and cycline E. ${ }^{35}$ It also prevents atrophic arthritis through apoptosis and inhibits the production of prostaglandin E2 in synovial fibroblasts in patients with atrophic arthritis. ${ }^{36,37}$ It checks allergic reactions by reducing Th2 inflammatory reactions. ${ }^{38}$ Cooper and Burton suggest that honey might reduce inflammation in an inflammatory site by quenching free radicals. ${ }^{16}$ Honey containing high levels of methylglyoxal (MGO), Chepulis and Francis reported that it had contradictory effects on neutrophil TNF- $\alpha$ production. ${ }^{39}$ Mathew et al. found that Spirulina fusiformis (1 g/day for 12 months) had chemo preventive activity in reversing oral leukoplakia in pan tobacco chewers in Kerala, India. ${ }^{40}$

\section{CONCLUSIONS}

The advantage of these natural products is the higher bioavailability, ease of use and most importantly lesser adverse effects. The present study was done to assess the combined immunomodulatory effect of garlic, turmeric, honey and spirulina using modern technique and a spectrophotometry was used for analysis. It showed significant antioxidant, immunomodulatory properties and higher zone of inhibition when compared to a standard. With more future studies, this extract can be used as an alternative to other commercially available drugs in the form of in situ gel for immune mediated mucocutaneous disorders.

\section{Acknowledgements}

The authors are grateful to Saveetha Institute of Medical and Technical Sciences for granting permission to conduct the present study and the faculties of Department of Oral Medicine for their critical review, support during the development and writing of these manuscripts.

Data sharing statement provided by the authors is available with the full text of this article at jemds.com.

Financial or other competing interests: None.

Disclosure forms provided by the authors are available with the full text of this article at jemds.com.

\section{REFERENCES}

[1] Catanzaro M, Corsini E, Rosini $M$, et al. Immunomodulators inspired by nature: a review on curcumin and echinacea. Molecules 2018;23(11):2778.

[2] Sell S. Immunology, Immunopathology and Immunity. $6^{\text {th }}$ edn. USA: American Society for Microbiology 2001. http://dx.doi.org/10.1128/9781555818012

[3] Fleit HB. Kuby Immunology. Goldsby RA, Kindt TJ, Osborne BA, eds. The Quarterly Review of Biology 2001;76:126.

[4] Houghton PJ, Mukherjee PK. Evaluation of herbal medicinal products: perspectives on quality, safety and efficacy. Pharmaceutical Press 2009: p. 502.

[5] Mukherjee PK, Venkatesh M, Gantait A. Ayurveda in modern medicine: development and modification of bioactivity. In: Comprehensive natural products chemistry. The Netherlands: Elsevier Publication 2009.

[6] Mukherjee PK, Nema NK, Venkatesh P, et al. Changing scenario for promotion and development of Ayurveda way forward. Journal of Ethnopharmacology 2012;143(2):424-34.

[7] Kyo E, Uda N, Kasuga S, et al. Immunomodulatory effects of aged garlic extract. J Nutr 2001;131(3s):1075S-9S.

[8] Arreola R, Quintero-Fabián S, López-Roa RI, et al. Immunomodulation and anti-inflammatory effects of garlic compounds. J Immunol Res 2015;2015:401630.

[9] Hodge G, Hodge S, Han P. Allium Sativum (garlic) suppresses leukocyte inflammatory cytokine production in vitro: potential therapeutic use in the treatment of inflammatory bowel disease. Cytometry 2002;48(4):20915.

[10] Bagad AS, Joseph JA, Bhaskaran N, et al. Comparative evaluation of anti-inflammatory activity of curcuminoids, turmerones and aqueous extract of curcuma longa. Adv Pharmacol Sci 2013;2013:805756.

[11] Rahmani AH, Alsahli MA, Aly SM, et al. Role of curcumin in disease prevention and treatment. Adv Biomed Res 2018;7:38

[12] Jagetia GC, Aggarwal BB. "Spicing up" of the immune system by curcumin. J Clin Immunol 2007;27(1):19-35. 
[13] Bose S, Panda AK, Mukherjee S, et al. Curcumin and tumor immune-editing: resurrecting the immune system. Cell Div 2015;10:6.

[14] Ranjan D, Chen C, Johnston TD, et al. Curcumin inhibits mitogen stimulated lymphocyte proliferation, NFkB activation and IL - 2 signaling. Journal of Surgical Research 2004;121(2):171-7.

[15] Yadav VS, Mishra KP, Singh DP, et al. Immunomodulatory effects of curcumin. Immunopharmacology and Immunotoxicology 2005;27(3):485-97.

[16] McLoone P, Warnock M, Fyfe L. Honey: an immunomodulatory agent for disorders of the skin. Food Agric Immunol 2016;27(3):338-49.

[17] Tonks AJ, Cooper RA, Jones KP, et al. Honey stimulates inflammatory cytokine production from monocytes. Cytokine 2003;21(5):242-7.

[18] Somchit MN, Mohamed NA, Ahmad Z, et al. Antiinflammatory and anti-pyretic properties of Spirulina platensis and Spirulina lonar: a comparative study. Pak J Pharm Sci 2014;27(5):1277-80.

[19] Soheili M, Khosravi-Darani K. The potential health benefits of algae and micro algae in medicine: a review on spirulina platensis. Curr Nutr Food Sci 2011;7(4):279-85.

[20] Finamore A, Palmery M, Bensehaila S, et al. Antioxidant, immunomodulating and microbial - modulating activities of the sustainable and ecofriendly spirulina. Oxid Med Cell Longev 2017;2017:3247528.

[21] Chen HW, Yang TS, Chen MJ, et al. Purification and immunomodulating activity of $\mathrm{C}$ - phycocyanin from Spirulina platensis cultured using power plant flue gas Process Biochemistry 2014;49(8):1337-44.

[22] Eilat-Adar S, Sinai T, Yosefy C, et al. Nutritional recommendations for cardiovascular disease prevention Nutrients 2013;5(9):3646-83.

[23] You S, Nakanishi E, Kuwata H, et al. Inhibitory effects and molecular mechanisms of garlic organosulfur compounds on the production of inflammatory mediators. Mol Nutr Food Res 2013;57(11):2049-60.

[24] Banchereau J, Briere F, Caux C, et al. Immunobiology of dendritic cells. Annual Review of Immunology 2000;18:767-811.

[25] Badr GM, Al-Mulhim JA. The protective effect of aged garlic extract on non-steroidal anti - inflammatory drug induced gastric inflammations in male albino rats. Evid Based Complement Alternat Med 2014;2014:759642.

[26] Lee W-H, Loo C-Y, Bebawy M, et al. Curcumin and its derivatives: their application in neuropharmacology and neuroscience in the 21st century. Curr Neuropharmacol 2013;11(4):338-78.
[27] Khar A, Ali AM, Pardhasaradhi BV, et al. Induction of stress response renders human tumor cell lines resistant to curcumin - mediated apoptosis: role of reactive oxygen intermediates. Cell Stress Chaperones 2001;6(4):368-76.

[28] Chan WH, Wu HY, Chang WH. Dosage effects of curcumin on cell death types in a human osteoblast cell line. Food and Chemical Toxicology 2006;44(8):1362-71.

[29] Limtrakul P. Curcumin as chemosensitizer. Advances in Experimental Medicine and Biology 2007;595:269-300.

[30] Natarajan C, Bright JJ. Curcumin inhibits experimental allergic encephalomyelitis by blocking IL - 12 signaling through Janus kinase - STAT pathway in T lymphocytes. J Immunol 2002;168(12):6506-13.

[31] Zhang L, Fiala M, Cashman J, et al. Curcuminoids enhance amyloid - beta uptake by macrophages of Alzheimer's disease patients. J Alzheimers Dis 2006;10(1):1-7.

[32] Chen HW, Kuo HT, Chai CY, et al. Pre-treatment of curcumin attenuates coagulopathy and renal injury in LPS - induced endotoxemia. J Endotoxin Res 2007;13(1):1523.

[33] Gradisar H, Keber MM, Pristovsek P, et al. MD - 2 as the target of curcumin in the inhibition of response to LPS. J Leukoc Biol 2007;82(4):968-74.

[34] Youn HS, Saitoh SI, Miyake K, et al. Inhibition of homodimerization of Toll - like receptor 4 by curcumin Biochemical Pharmacology 2006;72(1):62-9.

[35] Cho JW, Lee KS, Kim CW. Curcumin attenuates the expression of IL - $1 \beta$, IL - 6 and TNF - $\alpha$ as well as cyclin E in TNF - $\alpha$ - treated HaCaT cells; NF - карpaB and MAPKs as potential upstream targets. International Journal of Molecular Medicine 2007;19(3):469-74.

[36] Funk JL, Oyarzo JN, Frye JB, et al. Turmeric extracts containing curcuminoids prevent experimental rheumatoid arthritis. J Nat Prod 2006;69(3):351-5.

[37] Park C, Moon DO, Choi IW, et al. Curcumin induces apoptosis and inhibits prostaglandin E(2) production in synovial fibroblasts of patients with rheumatoid arthritis. Int J Mol Med 2007;20(3):365-72.

[38] Kurup VP, Barrios CS, Raju R, et al. Immune response modulation by curcumin in a latex allergy model Clinical and Molecular Allergy 2007;5:1.

[39] Chepulis LM, Francis E. An initial investigation into the anti - inflammatory activity and antioxidant capacity of alpha-cyclodextrin-complexed Manuka honey. J Complement Integr Med 2012; 9:Article 25.

[40] Mathew B, Sankaranarayanan R, Nair PP, et al. Evaluation of chemoprevention of oral cancer with spirulina fusiformis. Nutrition and Cancer 1995;24(2):197-202. 